IPM/P-2012/050

FPAUO-12/12

\title{
Charged Black Branes with Hyperscaling Violating Factor
}

\author{
Mohsen Alishahiha $^{a}$, Eoin Ó Colgáin ${ }^{b}$ and Hossein Yavartanoo $^{c}$ \\ ${ }^{a}$ School of Physics, Institute for Research in Fundamental Sciences (IPM) \\ P.O. Box 19395-5531, Tehran, Iran \\ ${ }^{b}$ Departamento de Física, Universidad de Oviedo, 33007 Oviedo, Spain \\ ${ }^{c}$ Department of Physics, Kyung-Hee University, Seoul 130-701, Korea
}

\begin{abstract}
We present an analytic solution of a charged black hole with hyperscaling violating factor in an Einstein-Maxwell-Dilaton model where the scalar potential is key to the existence of a solution. This solution provides a candidate gravitational description of theories with hyperscaling violation at both finite temperature and finite charge density. Using this background we explore certain features of these theories via AdS/CFT correspondence. Finally, we discuss embeddings based on the well-known sphere reductions of ten and eleven-dimensional supergravity.
\end{abstract}




\section{Introduction}

Gauge/gravity duality [1] may be thought of as a practical tool to study strongly-coupled systems near critical points where the system exhibits a scaling symmetry. Generally at a critical point the system may be described by a conformal field theory (CFT). From the gauge/gravity point of view this means that the gravitational theory is defined on a metric which is asymptotically locally Anti-de Sitter (AdS).

On the other hand, in many physical systems critical points are governed by dynamical scalings in which, even though the system exhibits a scaling symmetry, space and time scale differently under this symmetry. A prototype example of such critical points is a Lifshitz fixed point where the system is spatially isotropic and scale invariant, though there is an anisotropic scaling in the time direction characterized by a dynamical exponent, z. More precisely the system is invariant under the following scale symmetry

$$
r \rightarrow \lambda^{z} r, \quad x_{i} \rightarrow \lambda x_{i}
$$

where $t$ is time and $x_{i}$ are spatial coordinates of a $d$ dimensional space (in our case the $d$ dimensional space is $R^{d}$ ).

In the framework of gauge/gravity duality it is then important to find gravitational theories that provide a gravity description of Lifshitz fixed points. Indeed, such a gravity dual for the Lifshitz fixed point was introduced in [2] where the authors considered a gravitational theory which admits a solution exhibiting the above scaling symmetry (see also [3] for earlier work on a geometry with Lifshitz scaling.). The corresponding metric may be written as

$$
d s^{2}=-r^{2 z} d t^{2}+r^{2} \sum_{i=1}^{d} d x_{i}^{2}+\frac{d r^{2}}{r^{2}}
$$

In terms of bottom-up model building, a natural set-up to consider is Einstein-Maxwell-Dilaton (EMD) theory, a setting in which numerous studies of Lifshitz-like black brane geometries have already appeared [4 16]. As is well appreciated at this stage, with the inclusion of dilaton and Abelian gauge fields, it is possible to find even more sophisticated metrics generalizing Lifshitz which, on top of an anisotropic scaling, have also an overall hyperscaling factor. More precisely, one may have a geometry in the form of 6

$$
d s^{2}=r^{\frac{-2 \theta}{d}}\left(-r^{2 z} d t^{2}+r^{2} \sum_{i=1}^{d} d x_{i}^{2}+\frac{d r^{2}}{r^{2}}\right)
$$

where the constants $z$ and $\theta$ are dynamical and hyperscaling violation exponents, respectively. This geometry has been the subject of considerable recent attention and hyperscaling violating 
Lifshitz solutions have been found in a host of different settings $[17-22]$. This is the most general geometry which is spatially homogeneous and covariant under the following scale transformations

$$
t \rightarrow \lambda^{z} t, \quad r \rightarrow \lambda^{-1} r, \quad x_{i} \rightarrow \lambda x_{i}, \quad d s_{d+2} \rightarrow \lambda^{\frac{\theta}{d}} d s_{d+2} .
$$

Note that with a non-zero $\theta$, the distance is not invariant under the scaling which in the context of AdS/CFT indicates violations of hyperscaling in the dual field theory. More precisely, while in $(d+1)$-dimensional theories without hyperscaling, the entropy scales as $T^{d / z}$ with temperature in the present case where the metric exhibits hyperscaling, it scales as $T^{(d-\theta) / z}[23,24]$.

An interesting feature of the above metric is that for the special value of the hyperscaling violation exponent $\theta=d-1$, the holographic entanglement entropy 30, 31 exhibits a logarithmic violation of area law 24, 32,33, indicating that the background (1.3) could provide a gravitational dual for a theory with an $\mathcal{O}\left(N^{2}\right)$ Fermi surface, where $N$ is the number of degrees of freedom, or alternatively the number of colors for an $S U(N)$ gauge theory.

These observations indicate that backgrounds whose asymptotic behavior coincides with the above metric may be of interest to condensed matter physics, thus making it natural to further explore gauge/gravity duality for these backgrounds, some of the aspects of which have already been touched upon in 25,26]. As the eventual goal of this program is to make contact with condensed matter, a natural generalisation involves backgrounds with finite charge density. Recently, we have witnessed the first construction of finite temperature and finite charge density solutions in a top-down construction with probe D-branes [27]. Here, as a further step in this direction, we identify what we believe to be the first back-reacted hyperscaling violating backgrounds with nonzero temperature and charge. This extends hyperscaling geometries in EMD theories at finite temperature 24,25,28 to finite charge density.

This article is organized as follows. In the next section we will find hyperscaling violating charged black brane solutions in an EMD theory with a non-trivial potential for the dilaton. We observe that the presence and the form of the potential is crucial to have the solution. We shall also show that at zero temperature where the solution is extremal, the near-horizon geometry develops an $A d S_{2}$ factor indicating that theories with hyperscaling violation at finite charge density and zero temperature may be described by an IR fixed point. In section three we study holographic entanglement entropy for a hyperscaling violating black brane solution, where we explore how the non-zero charge affects the entanglement entropy. In section four we probe the background by a charged fermion where we investigate the possibility of having an $\mathcal{O}\left(N^{0}\right)$ Fermi surface in the model. In section five we study optical conductivity for theories with hyperscaling violation. The last section is devoted to discussions, in particular, those related to candidate embeddings.

Note added: While we were writing up we became aware of further Lifshitz solutions with hyperscaling violation [29]. 


\section{Gravity solution}

In this section we introduce our Einstein-Maxwell-Dilaton model and illustrate hyperscaling violating charged black branes for this theory. For the asymptotically Lifshitz geometry an instructive analytic solution for charged black branes has been found [14]. To find such a solution one needs at least two $U(1)$ gauge fields coupled to a scalar field. The first $U(1)$ together with the scalar field is necessary to generate an anisotropic scaling, while the second one is required to have a charged solution. It is also possible to find asymptotically charged black holes as well. For this purpose one should add another $U(1)$ gauge field to be responsible for the compacted horizon [14].

To find hyperscaling violating charged black branes we will closely follow the approach of [14]. We note, however, that in order to get a non-trivial hyperscaling factor, besides the action considered in [14], the model considered here has to have a non-trivial potential for the scalar field as well. In what follows we only consider the model with two $U(1)$ gauge fields and therefore we will find black branes charged under one $U(1)$ gauge field. We expect that it is straightforward to generalize for black holes with more gauge fields.

To proceed we consider a minimal model as follows

$$
S=-\frac{1}{16 \pi G} \int d^{d+2} x \sqrt{-g}\left[R-\frac{1}{2}(\partial \phi)^{2}+V(\phi)-\frac{1}{4} \sum_{1=1}^{2} e^{\lambda_{i} \phi} F_{i}^{2}\right]
$$

where we have not confined ourselves to any particular dimensionality. For the potential of the scalar field, motivated by the typical exponential potentials of string theory, we will consider the following potentia. $\bigsqcup^{1}$

$$
V=V_{0} e^{\gamma \phi}
$$

Here $\lambda_{1}, \lambda_{2}, \gamma$ and $V_{0}$ are free parameters of the model. We will see that with this simple potential, the model does, indeed, admit solutions with hyperscaling violation.

The equations of motion of the above action read

$$
\begin{aligned}
& R_{\mu \nu}+\frac{V(\phi)}{d} g_{\mu \nu}=\frac{1}{2} \partial_{\mu} \phi \partial_{\nu} \phi+\frac{1}{2} \sum_{i=1}^{2} e^{\lambda_{i} \phi}\left(F_{i \mu}^{\rho} F_{i \rho \nu}-\frac{g_{\mu \nu}}{2 d} F_{i}^{2}\right) \\
& \nabla^{2} \phi=-\frac{d V(\phi)}{d \phi}+\frac{1}{4} \sum_{i=1}^{2} \lambda_{i} e^{\lambda_{i} \phi} F_{i}^{2}, \quad \nabla_{\mu}\left(\sqrt{-g} e^{\lambda_{i} \phi} F_{i}^{\mu \nu}\right)=0
\end{aligned}
$$

Let us consider the following ansatz for the metric, scalar and gauge field

$$
d s^{2}=r^{2 \alpha}\left(-r^{2 z} f(r) d t^{2}+\frac{d r^{2}}{r^{2} f(r)}+r^{2} d \vec{x}^{2}\right), \quad \phi=\phi(r), \quad F_{i r t} \neq 0
$$

\footnotetext{
${ }^{1}$ Note that we do not expect to find this potential in string theory truncations of AdS vacua, but those of domain walls, i.e. the near-horizon of various branes.
} 
and assume that the other components of gauge fields are set to zero.

From the Maxwell equations of motion, using the above ansatz, one finds

$$
F_{i r t}=e^{-\lambda_{i} \phi} r^{\alpha(2-d)+z-d-1} \rho_{i}
$$

Then by combining the $t t$ and $r r$ components of the Einstein equation one has

$$
R_{t}^{t}-R_{r}^{r}=-\frac{1}{2} g^{r r}\left(\partial_{r} \phi\right)^{2}
$$

On the other hand, by calculating the Ricci tensor for our ansatz we get

$$
R_{t}^{t}-R_{r}^{r}=-d(\alpha+1)(\alpha+z-1) r^{-2 \alpha} f(r) .
$$

Plugging this expression back into the equation (2.6) one can determine scalar field as follows

$$
e^{\phi}=e^{\phi_{0}} r \sqrt{2 d(\alpha+1)(\alpha+z-1)}=e^{\phi_{0}} r^{\beta}
$$

Note that in order to have a well defined solution one has to assume $(\alpha+1)(\alpha+z-1) \geq 0$. Indeed it can be seen that this assumption is a consequence of the null energy condition. More precisely consider a null vector as 14$] \xi^{\mu}=\left(\sqrt{g^{r r}}, \sqrt{g^{t t}}, 0\right)$, then

$$
T_{\mu \nu} \xi^{\mu} \xi^{\nu} \sim R_{r}^{r}-R_{t}^{t}=d(\alpha+1)(\alpha+z-1) r^{-2 \alpha} f(r) \geq 0
$$

which in turn translates into $(\alpha+1)(\alpha+z-1) \geq 0$.

To find the metric one utilizes the other components of the Einstein equations. Indeed for our ansatz one has

$$
R_{x}^{x}=-(\alpha+1) r^{-\alpha(d+2)-z-d+1}\left(r^{d(\alpha+1)+z} f(r)\right)^{\prime},
$$

where, as in standard practice, a prime represents derivative with respect to $r$. Therefore the $x x$ component of the Einstein equations of motion reads

$$
\left(r^{d(\alpha+1)+z} f(r)\right)^{\prime}=\frac{r^{\alpha(d+2)+z+d-1}}{(\alpha+1)}\left(\frac{V_{0} e^{\gamma \phi}}{d}-\frac{1}{2 d} \sum_{i=1}^{2} e^{-\lambda_{i} \phi} \rho_{i}^{2} r^{-2 d(\alpha+1)}\right) .
$$

Using the solution of $\phi 2.8$ one arrives at

$$
\left(r^{d(\alpha+1)+z} f(r)\right)^{\prime}=\frac{r^{\alpha(d+2)+z+d-1}}{(\alpha+1)}\left(\frac{V_{0} e^{\gamma \phi_{0}} r^{\gamma \beta}}{d}-\frac{1}{2 d} \sum_{i=1}^{2} e^{-\lambda_{i} \phi_{0}} \rho_{i}^{2} r^{-2 d(\alpha+1)-\lambda_{i} \beta}\right)
$$


which can be integrated to find the function $f$ of the metric as follows

$$
\begin{aligned}
f(r)= & -m r^{-d \alpha-z-d}+\frac{V_{0} e^{\gamma \phi_{0}} r^{\gamma \beta+2 \alpha}}{d(\alpha+1)(\gamma \beta+\alpha(d+2)+z+d)} \\
& -\sum_{i=1}^{2} \rho_{i}^{2} e^{-\lambda_{i} \phi_{0}} \frac{r^{\left.-2 \alpha(d-1)-\beta \lambda_{i}-2 d\right)}}{2 d(\alpha+1)\left(\alpha(2-d)+z-d-\beta \lambda_{i}\right)},
\end{aligned}
$$

where $m$ is a constant which can be related to the mass of the black brane.

With this expression, we have found all functions involved in the ansatz (2.4). The resulting solution has seven free parameters: $\alpha, z, m, \phi_{0}, \rho_{1}, \rho_{2}$ and the radius of the metric which has already been set to unity. On the other hand the action has four free parameters $\lambda_{1}, \lambda_{2}, \gamma$ and $V_{0}$. Therefore four parameters of the solution can be fixed by the four parameters of the model yielding a solution with three parameters which correspond to the mass, charge and the value of the scalar field, let's say, at horizon.

Note that so far we have not used all equations of motion. Indeed, in order, to find the parameters of the solution in terms of those in the action, we now utilize the remaining equations of motion. To proceed, we note that in order to maintain the asymptotic behavior of the hyperscaling violating metric, as it is evident from the expression of $f$, one must fix $\gamma$ as follows

$$
\gamma=-\frac{2 \alpha}{\beta}
$$

From this expression it is clear that to find a charged black brane (hole) with hyperscaling violating factor, it is crucial to have a non-trivial potential.

From the equation of motion of the scalar field, we get

$$
\left(\frac{4 \beta}{d(\alpha+1)}-\frac{8 \alpha}{\beta}\right) V_{0} e^{-2 \alpha \phi / \beta}=\sum_{i=1}^{2} e^{\lambda_{i} \phi} F_{i}^{2}\left(\lambda_{i}-\frac{\beta}{d(\alpha+1)}\right)
$$

This equation can be solved for parameters $\lambda_{1}, \lambda_{2}$ and $\rho_{1}$. Actually this equation may be solved in different, but rather equivalent, ways. In particular one may find ${ }^{2}$

$$
\lambda_{1}=-\frac{2 \alpha(d-1)+2 d}{\sqrt{2 d(\alpha+1)(\alpha+z-1)}}, \quad \lambda_{2}=\sqrt{\frac{2(\alpha+z-1)}{d(\alpha+1)}}, \quad \rho_{1}^{2}=\frac{2 V_{0}(z-1) e^{-\sqrt{\frac{2 d(\alpha+1)}{\alpha+z-1}} \phi_{0}}}{d \alpha+d+z-1} .
$$

With these expressions, $\rho_{2}$ remains as an undetermined free parameter which, indeed, can be identified with the charge of the solution. The last parameter, $V_{0}$, may also be fixed by setting the

\footnotetext{
${ }^{2}$ It is worth mentioning that instead of finding the parameters of the solution in terms of those in action, we have fixed the action in terms of parameters of the solution.
} 
constant term in the expression of $f$ to one. Doing so, one arrives at

$$
V_{0}=e^{\frac{2 \alpha \phi_{0}}{\sqrt{2 d(1+\alpha)(-1+z+\alpha)}}}(d \alpha+z+d-1)(d \alpha+z+d)
$$

With this equation we have fixed all four free parameters, though there are still unused equations of motion. It is, then, important to check whether the other equations hold without imposing any further constraints on the parameters of the solution. In particular one of the non-trivial equations need to be checked is the $t t$ component of the Einstein equations of motion. Indeed it is easy to see that this equation is also satisfied without imposing any further constraints.

Therefore, to summarize, we note that the action (2.1) admits hyperscaling violating charged black brane solutions which can be recast to the following form ${ }^{3}$

$$
\begin{aligned}
d s^{2} & =r^{-2 \frac{\theta}{d}}\left(-r^{2 z} f(r) d t^{2}+\frac{d r^{2}}{r^{2} f(r)}+r^{2} d \vec{x}^{2}\right) \\
F_{1 r t} & =\sqrt{2(z-1)(z+d-\theta)} e^{\frac{\theta(1-d) / d+d}{\sqrt{2(d-\theta)(z-1-\theta / d)}} \phi_{0}} r^{d+z-\theta-1}, \\
F_{2 r t} & =Q \sqrt{2(d-\theta)(z-\theta+d-2)} e^{-\sqrt{\frac{z-1-\theta / d}{2(d-\theta)}} \phi_{0}} r^{-(z+d-\theta-1)}, \\
e^{\phi} & =e^{\phi_{0}} r^{\sqrt{2(d-\theta)(z-1-\theta / d)}}
\end{aligned}
$$

with

$$
f(r)=1-\frac{m}{r^{z+d-\theta}}+\frac{Q^{2}}{r^{2(z+d-\theta-1)}} .
$$

This is indeed a charged black brane solution whose radius of horizion, $r_{H}$, is obtained by setting $f=0$ which leads to the following algebraic equation for $r_{H}$ :

$$
r_{H}^{2(d+z-\theta-1)}-m r_{H}^{d+z-\theta-2}+Q^{2}=0 .
$$

The corresponding Hawking temperature is also found

$$
T=\frac{(d+z-\theta) r_{H}^{z}}{4 \pi}\left(1-\frac{(d+z-\theta-2) Q^{2}}{d+z-\theta} r_{H}^{2(\theta-d-z+1)}\right) .
$$

Therefore the extremal limit is given by

$$
r_{H}^{2(d+z-\theta-1)}=\frac{(d+z-\theta-2)}{d+z-\theta} Q^{2},
$$

\footnotetext{
${ }^{3}$ Note that in order to follow the standard notation in the literature we set $\alpha=-\theta / d$, where $\theta \geq 0$ is the hyperscaling violation exponent. Observe also that this solution is not valid for $\theta=d$ where $\alpha=-1$.

${ }^{4}$ As a consistency check, we note that for $d+z-\theta=2$, this solution reduces to an uncharged solution obtained in 25 .
} 
in which case the function $f$ reads

$$
f=1-\frac{2(d+z-\theta-1)}{d+z-\theta-2}\left(\frac{r_{H}}{r}\right)^{d+z-\theta}+\frac{d+z-\theta}{d+z-\theta-2}\left(\frac{r_{H}}{r}\right)^{2(d+z-\theta-1)} .
$$

In this limit it is easy to see that the solution near the horizon develops an $A d S_{2} \times R^{d-1}$ geometry. More precisely, in the extremal case one can consider the following change of coordinates

$$
r-r_{H}=\frac{\epsilon r_{H}^{2}}{(d+z-\theta)(d+z-\theta-1) \zeta}, \quad t=\frac{\tau}{\epsilon r_{H}^{z}}
$$

Then the near-horizon limit is defined by the limit $\epsilon \rightarrow 0$ for which the solution 2.18 reads

$$
\begin{aligned}
d s^{2} & =r_{H}^{2-2 \frac{\theta}{d}}\left(\frac{-d \tau^{2}+d \zeta^{2}}{(d+z-\theta)(d+z-\theta-1) \zeta^{2}}+d \vec{x}^{2}\right) \\
A_{1 \tau} & =\frac{\sqrt{2(z-1)(z+d-\theta)}}{(d+z-\theta)(d+z-\theta-1)} \frac{e^{\frac{\theta(1-d) / d+d}{\sqrt{2(d-\theta)(z-1-\theta / d)}} \phi_{0}}}{r_{H}^{\theta-d-1}} \frac{1}{\zeta}, \\
A_{2 \tau} & =\frac{Q \sqrt{2(d-\theta)(z-\theta+d-2)}}{(d+z-\theta)(d+z-\theta-1)} \frac{e^{-\sqrt{\frac{z-1-\theta / d}{2(d-\theta)}} \phi_{0}}}{r_{H}^{2 z+d-\theta-3}} \frac{1}{\zeta}, \\
e^{\phi} & =e^{\phi_{0}} r_{H}^{\sqrt{2(d-\theta)(z-1-\theta / d)}}
\end{aligned}
$$

which is $A d S_{2} \times R^{d}$ with two charges.

Following the general idea of gauge/gravity duality we would like to consider the solution (2.18) as a gravitational background dual to a theory with hyperscaling violation at finite temperature and finite charge density. We note that the gravitational description of theories with hyperscaling violation at finite temperature has already been studied in 25]. Actually the aim of the following sections is to further explore the holographic description of the theories with hyperscaling violation when the effect of charge has also been taken into account.

As an observation, we note that due to non-zero charge, the black brane could be extremal with near horizon of $A d S_{2}$ as we just demonstrated. It means that at low temperature the theory flows to an IR fixed point, where the theory may be governed by a two-dimensional CFT.

\section{Entanglement entropy}

The aim of this section is to study the holographic entanglement entropy of a hyperscaling violating charged black brane by making use of the AdS/CFT correspondence. To compute the entanglement entropy via AdS/CFT correspondence one needs to minimize a surface in the bulk gravity. More precisely, given a gravitational theory with the bulk Newton's constant $G_{N}$, the 
holographic entanglement entropy is given by 30,31

$$
S_{A}=\frac{\operatorname{Area}\left(\gamma_{A}\right)}{4 G_{N}}
$$

where $\gamma_{A}$ is the minimal surface in the bulk whose boundary coincides with the boundary of the entangling region.

In this section we will only consider the extremal case where the temperature is zero and therefore we will be able to study the effects of non-zero charge on the entanglement entropy. In the non-extremal case where we have non-zero temperature, the results essentially reduce to those in 25]. This is due to the fact that, in this case, the charge effects are subleading to the thermal behavior of the entanglement entropy.

To proceed, let is consider a long strip in the dual theory given by

$$
-\frac{\ell}{2} \leq x_{1} \leq \frac{\ell}{2}, \quad 0 \leq x_{i} \leq L \quad \text { for } i=2, \cdots, d
$$

The surface $\gamma_{A}$ is defined by a hypersurface whose boundary coincides with the above strip and has a profile in the bulk of the metric (2.25) given by $x_{1}=x(r)$. The induced metric on this hypersurface is

$$
d s_{\text {in }}^{2}=\rho^{2 \frac{\theta}{d}-2}\left[\left(\frac{1}{f(\rho)}+x^{\prime}(\rho)^{2}\right) d \rho^{2}+d x_{i}^{2}\right]
$$

where we have made a change of coordinate, $r=\frac{1}{\rho}$, and prime now represents the derivative with respect to $\rho$. Therefore the area of the hypersurface reads

$$
A=L^{d-1} \int d \rho \rho^{\theta-d} \sqrt{f^{-1}+x^{\prime 2}}
$$

As is well known, the above expression for the area may be treated as an action for a one dimensional mechanical system where the momentum conjugate of the field $x$ is conserved. So that

$$
\frac{x^{\prime}}{\sqrt{f^{-1}+x^{\prime 2}}}=\left(\frac{\rho_{0}}{\rho}\right)^{\theta-d}
$$

with $\rho_{0}$ representing the turning point where $x^{\prime}\left(\rho_{0}\right) \rightarrow \infty$. It is then easy to find the width of the strip as follows

$$
\ell=2 \rho_{0} \int_{0}^{1} \frac{\xi^{d-\theta} f^{-1 / 2}\left(\xi, \rho_{0} / \rho_{H}\right)}{\sqrt{1-\xi^{2(d-\theta)}}}
$$

where

$$
f\left(\xi, \frac{\rho_{0}}{\rho_{H}}\right)=1-\frac{2(d+z-\theta-1)}{d+z-\theta-2}\left(\frac{\rho_{0}}{\rho_{H}}\right)^{d+z-\theta} \xi^{d+z-\theta}+\frac{d+z-\theta}{d+z-\theta-2}\left(\frac{\rho_{0}}{\rho_{H}}\right)^{2(d+z-\theta-1)} \xi^{2(d+z-\theta-1)} .
$$


The area is then given by

$$
A=L^{d-1} \rho_{0}^{\theta-d+1} \int_{\frac{\epsilon}{\rho_{0}}}^{1} \frac{\xi^{\theta-d} f^{-1 / 2}\left(\xi, \rho_{0} / \rho_{H}\right)}{\sqrt{1-\xi^{2(d-\theta)}}}
$$

Now the aim is to perform the above integrals and eliminate $\rho_{0}$ to find the entanglement entropy as a function of the width of the strip $\ell$. Of course in general it cannot be done analytically, though in special limits it it possible to do so.

Let's assume that the width of strip is very small, which in turn results in the turning point being much smaller than the radius of the horizon, i.e. $\rho_{0} \ll \rho_{H}$. For this case when $\theta \neq d-1$, at leading order, the finite part of the entanglement entropy is

$$
S_{\text {finite }} \approx \frac{L^{d-1} \ell^{\theta-d+1}}{4 G_{N}}\left(1+a_{0}(z, d, \theta) Q^{\frac{d+z-\theta}{d+z-\theta-1}} \ell^{d+z-\theta}+a_{1}(z, d, \theta) Q^{2} \ell^{2(d+z-\theta-1)}\right)
$$

while for $\theta=d-1$ the entanglement entropy exhibits a logarithmic violation of area law

$$
S \approx \frac{L^{d-1}}{4 G_{N}} \ln \frac{\ell}{\epsilon}+c_{0}(z, d) Q^{\frac{z+1}{z}} \frac{L^{d-1} \ell^{z+1}}{4 G_{N} \epsilon^{z+1}}+c_{1}(d, z) Q^{2} \frac{L^{d-1} \ell^{2 z}}{4 G_{N} \epsilon^{2 z}} .
$$

This is in fact a signature that the dual theory has an $\mathcal{O}\left(N^{2}\right)$ Fermi surface which can be probed by the enanglement entropy 24,32 . Here $a_{i}(z, d, \theta)$ and $c_{i}(z, d)$ are numerical constants.

On the other hand if we want to consider a strip with large width, one should let the hypersurface extend all the way to the deep IR region, i.e. $\rho_{0} \sim \rho_{H}$. In this case, at leading order, one arrives at

$$
S_{\text {finite }} \approx \frac{L^{d-1} \ell}{4 G_{N}} Q^{\frac{d-\theta}{d+z-\theta-1}}
$$

It is worth mentioning that if we compute the Hawking entropy of the extremal charged black brane the resultant entropy is

$$
S_{\mathrm{H}} \sim \frac{\mathrm{Vol}_{d}}{4 G_{n}} Q^{\frac{d-\theta}{d+z-\theta-1}}
$$

which has the same behavior as the above entanglement entropy. Note that here $\mathrm{Vol}_{d}$ is the volume of $d$ dimensional space generated by $\vec{x}$.

\section{Charged Fermion probe}

In the previous section we have studied holographic entanglement entropy for theories with hyperscaling violation. If the theory has an $\mathcal{O}\left(N^{2}\right)$ Fermi surface, the holographic entanglement entropy is able to probe it. Indeed, in this case the entanglement entropy exhibits a logarithmic violation of the area law. Actually for the model we are studying when $\theta=d-1$ with or without charge, we have a logarithmic violation of area law, showing that it has an $\mathcal{O}\left(N^{2}\right)$ Fermi surface 
(see also $[24,32]$ ).

On the other hand when $\theta \neq d-1$, the entanglement entropy has a power law behavior which indicates that the dual theory does not have an $\mathcal{O}\left(N^{2}\right)$ Fermi surface. Nevertheless one may wonder whether the model still has an $\mathcal{O}\left(N^{0}\right)$ Fermi surface. Of course in this case the entanglement entropy cannot probe the Fermi surface. In fact, to see whether the system exhibits an $\mathcal{O}\left(N^{0}\right)$ Fermi surface one could probe the system by a charged fermion. Then to see a hint of a Fermi surface, one should look for a sharp behavior in the fermionic retarded Green's function at finite momentum and small frequencies [39 45] (for a review see [46]).

In this section we will probe the extremal hyperscaling violating black brane geometry by a charged fermion!5. This can be used to read off the retarded Green's function of a fermionic operator in the dual theory, which is a theory with hyperscaling violation at finite density. Using the pole structure of the corresponding retarded Green's function, we gain an insight into whether, or not, a Fermi surface exists. Since we will have to deal with fermions, for simplicity, we will only consider the four dimensional $(d=2)$ case. It is, of course, straightforward to generalize our analysis here to arbitrary dimensions.

To proceed, let us consider a four dimensional charged Dirac fermion on the background (2.18) in the extremal limit

$$
S_{\text {bulk }}=\int d^{4} x \sqrt{-g} i \bar{\Psi}\left[\frac{1}{2}\left(\Gamma^{a} \vec{D}_{a}-\overleftarrow{D}_{a} \Gamma^{a}\right)-m\right] \Psi
$$

Here $\Gamma^{a} D_{a}=\left(e_{\mu}\right)^{a} \Gamma^{\mu}\left[\partial_{a}+\frac{1}{4}\left(\omega_{\rho \sigma}\right)_{a} \Gamma^{\rho \sigma}-i q A_{2 a}\right]$, with $\Gamma^{\mu \nu}=\frac{1}{2}\left[\Gamma^{\mu}, \Gamma^{\nu}\right]$.

It is important to note that in order to find the equations of motion for this action, one needs to use the variational principle, which for a space with a boundary, always comes with a proper boundary condition. Of course, the boundary term is not necessarily unique and, indeed, for the above fermion action it was shown in 47] that there are numerous ways to make use of different boundary terms to make the variational principle well-defined. Of course, a given boundary term may break the symmetries of the model. In what follows we will consider the standard boundary condition (in the notation of 47$]$ ). The equation of motion 6

$$
\left(\left(e_{\mu}\right)^{a} \Gamma^{\mu}\left[\partial_{a}+\frac{1}{4}\left(\omega_{\rho \sigma}\right)_{a} \Gamma^{\rho \sigma}-i q A_{2 a}\right]-m\right) \Psi=0
$$

Here the non-zero components of the vielbeins and spin connections for the metric in equation (2.18) are

$$
\left(e_{t}\right)^{a}=\frac{r^{\theta / 2-z}}{\sqrt{f}} \delta_{t}^{a}, \quad\left(e_{r}\right)^{a}=r^{\theta / 2+1} \sqrt{f} \delta_{t}^{a}, \quad\left(e_{i}\right)^{a}=r^{\theta / 2-1} \delta_{i}^{a},
$$

\footnotetext{
${ }^{5}$ Fermions in asymptotically Lifshitz geometries have been sudied in 3438 .

${ }^{6}$ As it is evident fron our solution, the background is charged under the second gauge field and the first gauge is present to provide an anisotropic scaling along the time direction. Therefore in what follows we consider a fermion which is charged under the second gauge field.
} 
and

$$
\left(\omega_{t r}\right)_{a}=-\frac{\left(r^{2 z-\theta}\right)^{\prime}}{\left.2 r^{(} z-1-\theta\right)} \delta_{a t}, \quad\left(\omega_{i r}\right)_{a}=\frac{2-\theta}{2} r \sqrt{f} \delta_{a i}
$$

Then through the choice of $\Psi=\left(-g g^{r r}\right)^{-1 / 4} e^{-i \omega t+i k \cdot x} \psi(r)$ with $g$ being the determinant of the metric, the above equation of motion reduces to ${ }^{7}$

$$
\left[r f^{1 / 2} \Gamma^{r} \partial_{r}-\frac{i}{r^{z} f^{1 / 2}}\left(\omega+q \mu_{2}\left(1-\frac{1}{r^{z-\theta}}\right)\right) \Gamma^{t}+\frac{i}{r} \Gamma \cdot k-\frac{m}{r^{\theta / 2}}\right] \psi(r)=0,
$$

where $\mu_{2}=Q \sqrt{\frac{2(2-\theta)}{z-\theta}} e^{-\sqrt{\frac{2 z-2-\theta}{4(z-\theta)}} \phi_{0}}$. Note that we have normalized the gauge field in such a way that it vanishes at the horizon.

To find the retarded Green's function, we will utilize a numerical method to solve the equation of motion. To proceed, it is useful to consider the following representation for the four dimensional gamma matrices

$$
\Gamma^{r}=\left(\begin{array}{cc}
-\sigma^{3} & 0 \\
0 & -\sigma^{3}
\end{array}\right), \Gamma^{t}=\left(\begin{array}{cc}
i \sigma^{1} & 0 \\
0 & i \sigma^{1}
\end{array}\right), \Gamma^{1}=\left(\begin{array}{cc}
-\sigma^{2} & 0 \\
0 & \sigma^{2}
\end{array}\right), \Gamma^{2}=\left(\begin{array}{cc}
0 & -i \sigma^{2} \\
i \sigma^{2} & 0
\end{array}\right)
$$

Due to rotational symmetry in the spatial directions we may set $k_{2}=0$. Then using the notation

$$
\psi=\left(\begin{array}{l}
\Phi_{1} \\
\Phi_{2}
\end{array}\right)
$$

the equation of motion 4.5 reduces to the following decoupled equations

$$
\left[r f^{1 / 2} \partial_{r}-\frac{1}{r^{z} f^{1 / 2}}\left(\omega+q \mu_{2}\left(1-\frac{1}{r^{z-\theta}}\right)\right) i \sigma^{2}+\frac{m}{r^{\theta / 2}} \sigma^{3}-(-1)^{\alpha} \frac{k_{1}}{r} \sigma^{1}\right] \Phi_{\alpha}=0
$$

for $\alpha=1,2$. It is easy to see that

$$
\Phi_{\alpha} \sim a_{\alpha}\left(\begin{array}{l}
0 \\
1
\end{array}\right)+b_{\alpha}\left(\begin{array}{l}
1 \\
0
\end{array}\right), \quad \text { for } r \rightarrow \infty
$$

Note that the asymptotic behavior of different components of the fermions are independent of $m$. This is in contrast to the Lifshitz case where we have 36

$$
\Phi_{\alpha} \sim a_{\alpha} r^{m}\left(\begin{array}{l}
0 \\
1
\end{array}\right)+b_{\alpha} r^{-m}\left(\begin{array}{l}
1 \\
0
\end{array}\right), \quad \text { for } r \rightarrow \infty .
$$

Actually, this means that in our case we can use both standard and alternative quantization to read the retarded Green's function. In other words, one may consider either of $a_{\alpha}$ or $b_{\alpha}$ as the

\footnotetext{
${ }^{7}$ Here we have set the radius of the horizon to one.
} 
source and the other as the response.

To find the retarded Green's function, following [42], it is useful to set $\chi_{1}=\psi_{1} / \psi_{2}$ and $\chi_{2}=$ $\psi_{3} / \psi_{4}$ where $\psi_{i}$ 's are defined via $\Phi_{1}=\left(\psi_{1}, \psi_{2}\right), \Phi_{2}=\left(\psi_{3}, \psi_{4}\right)$. These parameters satisfy the following equations

$$
\begin{aligned}
& r f^{1 / 2} \partial_{r} \chi_{1}+\frac{2 m}{r^{\theta / 2}} \chi_{1}-\left(\frac{\Omega}{r^{z} f^{1 / 2}}+\frac{k_{1}}{r}\right) \chi_{1}^{2}=\frac{\Omega}{r^{z} f^{1 / 2}}-\frac{k_{1}}{r}, \\
& r f^{1 / 2} \partial_{r} \chi_{2}+\frac{2 m}{r^{\theta / 2}} \chi_{2}-\left(\frac{\Omega}{r^{z} f^{1 / 2}}-\frac{k_{1}}{r}\right) \chi_{2}^{2}=\frac{\Omega}{r^{z} f^{1 / 2}}+\frac{k_{1}}{r},
\end{aligned}
$$

where

$$
\Omega=\omega+q \mu_{2}\left(1-\frac{1}{r^{z-\theta}}\right) .
$$

Using these equations the retarded Green's function is essentially given in terms of functions $G_{1}(k, \omega)$ and $G_{2}(k, \omega)$ where 8

$$
G_{\alpha}(k, \omega)=\lim _{r \rightarrow \infty} \chi_{\alpha}, \quad \text { for } \alpha=1,2
$$

with the ingoing boundary condition imposed at the horizon, which in our notation is 42

$$
\left.\chi_{\alpha}\right|_{\text {horizon }}=i \text {. }
$$

More concretely, the corresponding retarded Green's function may be given by

$$
G(k, \omega)=-\left(\begin{array}{cc}
G_{1}(k, \omega) & 0 \\
0 & G_{2}(k, \omega)
\end{array}\right) .
$$

Therefore the spectral function reads

$$
\mathcal{A}(k, \omega)=\frac{1}{\pi} \operatorname{Im}\left(G_{1}(k, \omega)+G_{2}(k, \omega)\right) .
$$

Having found expressions for the retarded Green's function, it is an easy task to further determine its behavior as a function of $k$ and $\omega$. We have illustrated the behavior in figure 1 for $m=0, q \mu_{2}=$ $\sqrt{3}$.

We have plotted the spectral function for $\theta=1, z=2$ and $\theta=0.4, z=1.4$. To compare the results with those in the RN AdS case studied in [42], we have also plotted this case as well. Note that in all cases we have $z-\theta=1$. Observe that since the retarded Green's function is an even function of $k$ we only considered $k>0$.

One observes that the effect of increasing $\theta$ or $z$, is to broaden the sharp peak representing the

\footnotetext{
${ }^{8}$ Here we set $k_{1}=k$.
} 

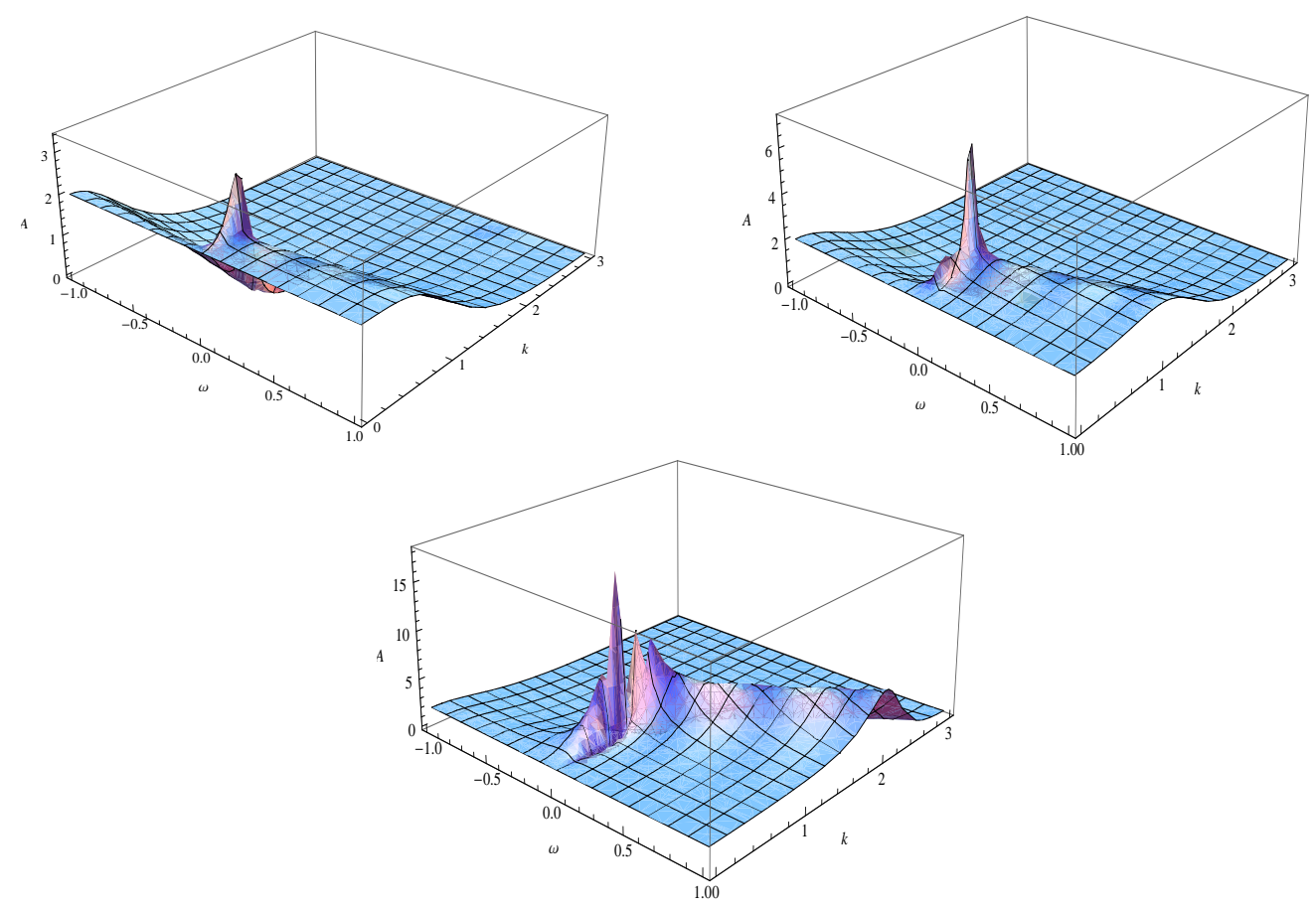

Figure 1: The behavior of spectral functions the for hyperscaling violating geometry with $\theta=$ $1, z=2$ (left), and $\theta=0.4, z=1.4$ (right). To compare the results with that of RN AdS black brane we have also plotted this case which in our notation corresponds to $\theta=0, z=1$ (see [42]).

$\mathcal{O}\left(N^{0}\right)$ Fermi surface. Moreover, as we see, even though for $\theta=0.4, z=1.4$ the entanglement entropy does not exhibit a violation of area law, at the probe limit there is almost a sharp peak in the fermionic retarded Green's function which might indicate that the system has a Fermi surface.

For $z=2$ and $\theta=1$, where the entanglement entropy exhibits a violation of area law indicating a Fermi surface at $\mathcal{O}\left(N^{2}\right)$, we still have a peak in the spectral function around $\omega \approx 0$, though it is not as sharp as that in the RN AdS black brane.

\section{Optical conductivity}

In this section we study the optical conductivity of a finite density system whose gravity dual is given by (2.18). The local $U(1)$ gauge field in the bulk is dual to a conserved current of a global $U(1)$ symmetry in the dual theory. In terms of the conserved current, the optical conductivity can be obtained from the Kubo formula as follows

$$
\sigma(\omega)=\frac{1}{i \omega}\langle J(\omega) J(-\omega)\rangle_{\mathrm{retarded}},
$$

where $J(\omega)$ is the conserved current of the $U(1)$ global symmetry evaluated at zero spatial momentum. The right hand side can be calculated using the AdS/CFT correspondence. Since we are working in the classical gravity regime, the leading contribution of the classical fluctuations of 
the gauge field is of $\mathcal{O}\left(N^{2}\right)$ which is due to the black brane background. In particular, it is then interesting to determine the optical conductivity for the case of $\theta=d-1$ where we have an $\mathcal{O}\left(N^{2}\right)$ Fermi surface.

To proceed, we consider small fluctuations of the gauge field in the $x_{1}$-direction, $\delta A_{x_{1}} \equiv a_{1}$. In general, turning on small fluctuations of the gauge field causes a back-reaction on the other fields of the model, so we have to solve the equations of motion for all the fluctuations. More precisely one may have

$$
A_{2 \mu} \rightarrow A_{2 \mu} \delta_{\mu 0}+a_{\mu}, \quad g_{\mu \nu} \rightarrow g_{\mu \nu}+h_{\mu \nu}, \quad \phi \rightarrow \phi+\delta \phi
$$

Since we are interested in calculating the two point function, it is enough to expand the action up to quadratic level in the fluctuations. To do so, it is convenient to first use the gauge freedom and set $h_{r \mu}=a_{r}=0$. Moreover at the level we are interested in we may set $\delta \phi=0$. With these assumptions it is straightforward to compute the quadratic terms of the action from which we can read off the equations of motion for the fluctuations. For our purpose, we will focus on the zero momentum case which means we may set $a_{1}=a(r) e^{-i \omega t}$. In this case the gauge field fluctuations mix only with the $h_{t x}$ component of the metric fluctuations. Therefore we find two coupled differential equations. Nevertheless one may eliminate $h_{t x}$ from the equations leading to the following differential equation for the gauge fluctuation: 9

$$
\partial_{r}\left[\sqrt{-g} e^{\lambda_{2} \phi} g^{r r} g^{x x} a^{\prime}(r)\right]+\left(\frac{\mathcal{Q}^{2} g_{r r} g_{t t}}{\sqrt{-g} g_{x x}}-\omega^{2} \sqrt{-g} g^{t t} g^{x x} e^{\lambda_{2} \phi}\right) a(r)=0,
$$

where $\mathcal{Q}=\sqrt{-g} e^{\lambda_{2} \phi} g^{t t} g^{r r} F_{2 r t}$. For the solution 2.18 the above equation reads ${ }^{10}$

$$
\partial_{r}\left[r^{3(z-1)+d-\theta} f a^{\prime}(r)\right]-\left[2 Q^{2}(d-\theta)(z-\theta+d-2) r^{z-3+\theta-d}-\frac{\omega^{2} r^{z-\theta+d-5}}{f}\right] a(r)=0 .
$$

Then for the special case of $\theta=d-1$ the equation reduces to

$$
\partial_{r}\left[r^{3 z-2} f a^{\prime}(r)\right]-\left[2 Q^{2}(z-1) r^{z-4}-\frac{\omega^{2} r^{z-4}}{f}\right] a(r)=0, \text { with } f=1-\left(1+\frac{Q^{2}}{r_{H}^{2 z}}\right)\left(\frac{r_{H}}{r}\right)^{z+1}+\frac{Q^{2}}{r^{2 z}} \text {. }
$$

It is interesting to note that the dependence on $\theta$ and $d$ drop out from the equation and it simply depends on a given $z$.

Now the aim is to solve the above equation to find a solution with an ingoing boundary condition at the horizon. Then the corresponding retarded Green's function can be read from the asymptotic

\footnotetext{
${ }^{9}$ For more details see for example [50] and also Appendix 6.B of 51].

${ }^{10}$ Note that in what follows, for simplicity, we have set $\phi_{0}=0$.
} 

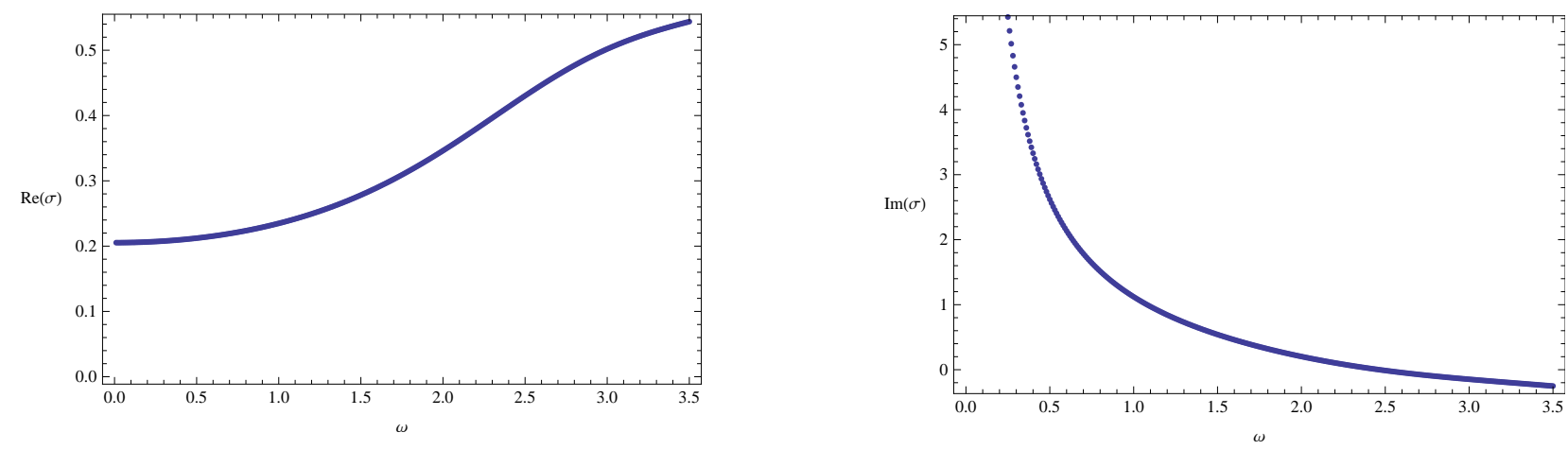

Figure 2: The real and imaginary parts of the conductivity versus $\omega$ for a fixed temperature. To find this plot we have set $Q=\sqrt{3}$, and $r_{H}=1.25$.

behavior of the solution near the boundary. Indeed one finds

$$
a \sim A+\frac{B}{r^{3(z-1)}}
$$

for $r \rightarrow \infty$ and at the horizon we get

$$
a \sim\left(r-r_{h}\right)^{ \pm i \frac{\omega^{2} r_{H}^{z}}{(z+1) r_{H}^{2 z}-Q^{2}(z-1)}}
$$

Therefore with a proper choice of the solution near the horizon (ingoing solution) the retarded Green's function is given by

$$
\left\langle J_{1}(\omega) J_{1}(-\omega)\right\rangle_{\text {retarded }} \sim \frac{B}{A} .
$$

In order to find the retarded Green's function we utilize numerical methods. To proceed, we note that the equation (5.5) has four free parameters, $Q, \omega, z$ and $r_{H}$. In fact setting $z=2$, and using "NDSolve" in Mathematica the real and imaginary parts of the conductivity as functions of frequency at a fixed temperature may be found as depicted in figure 2 .

The real part of the conductivity has the typical shape and from the imaginary part of the conductivity we observe that there is a delta function behavior in the real part. It is worth noting that the delta function behavior is an artifact of simplifications in the gravity calculation which should be compared with a sample without impurities. Adding impurities would broaden the delta function into a Drude peak. From the gravity point of view, this can be done by imposing a nontrivial boundary condition on the source of an operator at the boundary so that the translational invariance is broken. In this case, the Drude peak will appear from gravity calculations as well [52].

On the other hand, for a fixed $\omega$ one can find the behavior of the conductivity as a function of temperature. In particular, setting $\omega=0.001$, the real part of the conductivity can be found numerically as shown in figure 3. 


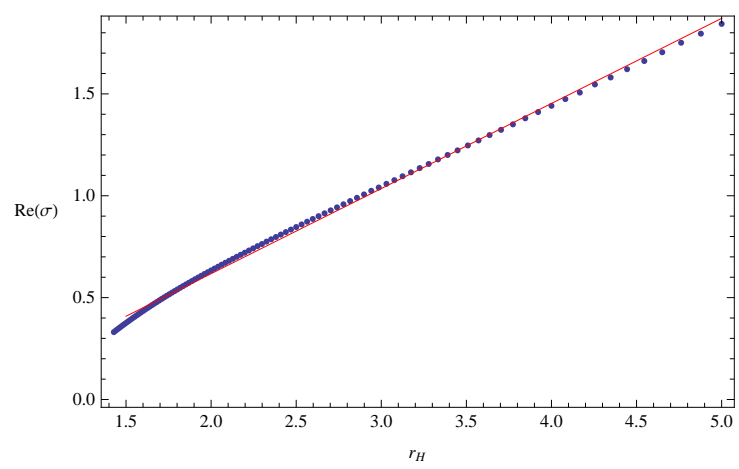

Figure 3: The real part of the conductivity versus $r_{H}$ for a fixed frequency. To find this plot we have set $Q=\sqrt{3}$, and $\omega=0.001$. The numerical solution is shown by dots, while the best fit is drawn by a red line.

Again using numerical methods the best fit one finds for the real part of the conductivity is

$$
\operatorname{Re}(\sigma) \approx-0.22+0.42 r_{H}
$$

Of course one may use the expression of the Hawking tempereture to write the result in terms of $T$. Indeed, doing so, one finds $\operatorname{Re}(\sigma) \approx T^{1 / 2}$.

\section{Discussions}

In this paper we have considered an EMD theory with a non-trivial potential for the dilaton. We have found an analytic solution representing a hyperscaling violating charged black brane solution. To do so, we have observed that the minimal model should contain at least two gauge fields, one to produce an anisotropic scaling and the other to correspond to a charge. It is known that to generate the required Lifshitz anisotropy, the first gauge field always diverges at the boundary, while the second falls off as a normal gauge field. Although in this paper we have only considered black branes with only one charge, we stress that generalizations to black holes with more charges are expected to be straightforward 14 .

Following the general thrust of the gauge/gravity philosophy, the resulting background (2.18) may provide gravity duals for strongly coupled theories with hyperscaling violation at finite charge density and temperature. It is then natural to explore different aspects of the theory by making use of gauge/gravity duality. Note that in order to study the effect of the charge, we have only considered the extremal case. Of course, one could have repeated the analysis for non-zero temperature, though the results at leading order would be the same as those obtained in [25]. This is because the effects associated to the charge are subleading. This can be seen, for example, in the expression of the temperature when expressed in terms of radius of the horizon. Moreover, the 
entropy of the black branes 2.18) at leading order in charge is given by

$$
S \sim T^{\frac{d-\theta}{z}}\left[1+\frac{(d-\theta)(d+z-\theta-2)}{z(d+z-\theta)} Q^{2} T^{\frac{2(\theta+1-d-z)}{z}}\right] .
$$

An immediate observation is that in order to have positive specific heat, at leading order we find the constraint $d \geq \theta$ [25], which was our assumption throughout the present paper.

We have used the extremal solution 2.25 to compute the entanglement entropy for a strip and we have been able to obtain the leading order correction to the entanglement entropy due to nonzero charge. We have observed that, even with non-zero charge, when $\theta=d-1$, the entanglement entropy, at leading order, exhibits a logarithmic violation of area law indicating that the dual theory may have an $\mathcal{O}\left(N^{2}\right)$ Fermi surface ${ }^{11}$. This, of course, would not exclude the possibility of having a Fermi surface of the order of one for arbitrary values of $\theta$.

Indeed, in order to study an order one Fermi surface we have also to probe the extremal background with a charged fermion. In the process, we have shown the retarded Green's function of the dual fermionic operator may have a pole in terms of a momentum for $\omega \approx 0$ indicating that there is the Fermi surface. We note, however, that as one increases $\theta$ or $z$, the sharp peak becomes smooth and eventually, for large enough $\theta$ and/or $z$, the peak disappears, suggesting that the system does not have a Fermi surface.

We have also studied optical conductivity at $\mathcal{O}\left(N^{2}\right)$ order numerically for the case of $\theta=d-1$ and $z=2$. We have found that in this case the real part of the conductivity, at leading order in charge, goes as $T^{1 / 2}$. Indeed, we could have done it for arbitrary $\theta, d$ and $z$. In this case one finds

$$
\operatorname{Re}(\sigma) \sim T^{\frac{d-\theta}{z}}
$$

As we have already mentioned, at zero temperature the geometry will develop an $A d S_{2}$ geometry with a non-zero gauge field at the near-horizon limit. This indicates that at low energy the theory is described by a two-dimensional CFT, which is dual to the $A d S_{2}$ geometry. However, due to the non-zero gauge field, the theory becomes unstable for sufficiently large charges. This is due to the fact that the scaling dimensions of operators in the dual theory become imaginary. If the operator is bosonic we would have bose condensation, while for a fermionic operator it may back-react to modify the geometry. Basically, the situation is very similar to that in a RN black hole [46].

It is also important to note that due to the hyperscaling violation and the behavior of the dilaton field at large distances, the background (1.3) cannot provide a dual description of a theory in all range of energies from UV to IR [48,49] (see also [25]). In fact, gravity on the background (2.18) may be considered as an effective theory which is valid over an intermediate energy scale.

What about string theory embeddings? We respond by echoing the sentiment of others by saying that it would be interesting to see if one can embed our solution to string theory. Here, to

\footnotetext{
${ }^{11}$ Another interesting observation is the charge dependence of the corrections is non-analytic.
} 
highlight that we do not expect this to be easy, we add some comments about embeddings based on the well-known sphere-reductions of ten and eleven-dimensional supergravity. In particular, we will focus on the truncations to $U(1)^{2}, U(1)^{3}$ and $U(1)^{4}$ gauged supergravities without axions which exist in in seven, five and four-dimensions respectively [53]. Starting with $d=5$ in our notation, neglecting the obvious drawback of it having a relatively more complicated potential, the $U(1)^{2}$ seven-dimensional gauged supergravity cannot be truncated further to a single scalar while retaining two independent gauge fields, so we can quickly eliminate this case.

The potentials for $d=2,3$ are both of the form

$$
V(\phi)=V_{1} e^{\gamma_{1} \phi}+V_{2} e^{\gamma_{2} \phi}
$$

and further truncations to a single scalar and two gauge fields can be found. So now we can return to section 2 and generalize the potential by adding the required extra exponential term. However, for these embeddings, if we require that one of the $\rho_{i}$ of our ansatz is a free parameter, such that it corresponds to the charge of a black hole, we find that the only solution is $\alpha=-1$, which we have already excluded from our solutions.

So, the simplest truncations based on sphere-reductions do not allow for embeddings. Given that we initially only considered an exponential potential, it seems promising that one could embed this using a domain wall type set-up, since such simple potentials typically arise there. However, we still require the exact field content of a single scalar and two gauge fields. We leave further exploration to future work.

\section{Acknowledgments}

We are grateful to Patrick Meessen and Nilanjan Sircar for discussion on related topics. M. A would like to thank TH-divsion of CERN where this work has done for very warm hospitality. The research

of M.A. is supported by Iran National Science Foundation (INSF). E. Ó C is partially supported by the research grants MICINN-09-FPA2009-07122 and MEC-DGI-CSD2007-00042. The work of H.Y is supported by the National Research Foundation of Korea Grant funded by the Korean Government (NRF-2011- 0023230).

\section{References}

[1] J. M. Maldacena, "The large N limit of superconformal field theories and supergravity," Adv. Theor. Math. Phys. 2, 231 (1998) [Int. J. Theor. Phys. 38, 1113 (1999)] hep-th/9711200. 
[2] S. Kachru, X. Liu and M. Mulligan, "Gravity duals of Lifshitz-like Fixed Points," Phys. Rev. D78 (2008) 106005, arXiv:0808.1725.

[3] P. Koroteev and M. Libanov, "On Existence of Self-Tuning Solutions in Static Braneworlds without Singularities," JHEP 0802, 104 (2008) [arXiv:0712.1136 [hep-th]].

[4] K. Goldstein, S. Kachru, S. Prakash and S. P. Trivedi, "Holography of Charged Dilaton Black Holes," JHEP 1008, 078 (2010) arXiv:0911.3586 [hep-th]].

[5] M. Cadoni, G. D’Appollonio and P. Pani, "Phase transitions between Reissner-Nordstrom and dilatonic black holes in 4D AdS spacetime," JHEP 1003, 100 (2010) arXiv:0912.3520 [hep-th]].

[6] C. Charmousis, B. Gouteraux, B. S. Kim, E. Kiritsis and R. Meyer, "Effective Holographic Theories for low-temperature condensed matter systems," JHEP 1011, 151 (2010) arXiv:1005.4690 [hep-th]].

[7] E. Perlmutter, "Domain Wall Holography for Finite Temperature Scaling Solutions," JHEP 1102, 013 (2011) |arXiv:1006.2124 [hep-th]].

[8] K. Goldstein, N. Iizuka, S. Kachru, S. Prakash, S. P. Trivedi and A. Westphal, "Holography of Dyonic Dilaton Black Branes," JHEP 1010, 027 (2010) arXiv:1007.2490 [hep-th]].

[9] G. Bertoldi, B. A. Burrington and A. W. Peet, "Thermal behavior of charged dilatonic black branes in AdS and UV completions of Lifshitz-like geometries," Phys. Rev. D 82, 106013 (2010) arXiv:1007.1464 [hep-th]].

[10] G. Bertoldi, B. A. Burrington, A. W. Peet and I. G. Zadeh, "Lifshitz-like black brane thermodynamics in higher dimensions," Phys. Rev. D 83, 126006 (2011) arXiv:1101.1980 [hep-th]].

[11] M. Cadoni and P. Pani, "Holography of charged dilatonic black branes at finite temperature," JHEP 1104, 049 (2011) arXiv:1102.3820 [hep-th]].

[12] N. Iizuka, N. Kundu, P. Narayan and S. P. Trivedi, "Holographic Fermi and Non-Fermi Liquids with Transitions in Dilaton Gravity," arXiv:1105.1162 [hep-th].

[13] P. Berglund, J. Bhattacharyya and D. Mattingly, "Charged Dilatonic AdS Black Branes in Arbitrary Dimensions," arXiv:1107.3096 [hep-th].

[14] J. Tarrio and S. Vandoren, "Black holes and black branes in Lifshitz spacetimes," JHEP 1109, 017 (2011) arXiv:1105.6335 [hep-th]].

[15] Y. S. Myung and T. Moon, "Quasinormal frequencies and thermodynamic quantities for the Lifshitz black holes," Phys. Rev. D 86, 024006 (2012) arXiv:1204.2116 [hep-th]]. 
[16] S. S. Pal, "Fermi-like Liquid From Einstein-DBI-Dilaton System," arXiv:1209.3559 [hep-th].

[17] H. Singh, "Special limits and non-relativistic solutions," JHEP 1012, 061 (2010) arXiv:1009.0651 [hep-th]].

[18] K. Narayan, "On Lifshitz scaling and hyperscaling violation in string theory," Phys. Rev. D 85, 106006 (2012) arXiv:1202.5935 [hep-th]].

[19] H. Singh, "Lifshitz/Schródinger Dp-branes and dynamical exponents," JHEP 1207, 082 (2012) arXiv:1202.6533 [hep-th]].

[20] P. Dey and S. Roy, "Lifshitz-like space-time from intersecting branes in string/M theory," arXiv:1203.5381 [hep-th].

[21] P. Dey and S. Roy, "Intersecting D-branes and Lifshitz-like space-time," arXiv:1204.4858 [hep-th].

[22] P. Dey and S. Roy, "Lifshitz metric with hyperscaling violation from NS5-Dp states in string theory," arXiv:1209.1049 [hep-th].

[23] B. Gouteraux and E. Kiritsis, "Generalized Holographic Quantum Criticality at Finite Density," JHEP 1112 (2011) 036 arXiv:1107.2116 [hep-th]].

[24] L. Huijse, S. Sachdev and B. Swingle, "Hidden Fermi surfaces in compressible states of gaugegravity duality," arXiv:1112.0573 [cond-mat.str-el].

[25] X. Dong, S. Harrison, S. Kachru, G. Torroba and H. Wang, "Aspects of holography for theories with hyperscaling violation," JHEP 1206, 041 (2012) arXiv:1201.1905 [hep-th]].

[26] M. Alishahiha and H. Yavartanoo, "On Holography with Hyperscaling Violation," arXiv:1208.6197 [hep-th].

[27] M. Ammon, M. Kaminski and A. Karch, "Hyperscaling-Violation on Probe D-Branes," arXiv:1207.1726 [hep-th].

[28] M. Cadoni and S. Mignemi, "Phase transition and hyperscaling violation for scalar Black Branes," JHEP 1206, 056 (2012) arXiv:1205.0412 [hep-th]].

[29] P. Bueno, W. Chemissany, P. Meessen, T. Ortin and C. S. Shahbazi, "Lifshitz-like solutions with hyperscaling violation in ungauged supergravity," arXiv:1209.4047 [hep-th].

[30] S. Ryu and T. Takayanagi, "Holographic Derivation of Entanglement Entropy from AdS/CFT," Phys. Rev. Lett. 96 (2006) 181602 [hep-th/0603001]. 
[31] S. Ryu and T. Takayanagi, "Aspects of Holographic Entanglement Entropy," JHEP 0608 (2006) 045 hep-th/0605073.

[32] N. Ogawa, T. Takayanagi and T. Ugajin, "Holographic Fermi Surfaces and Entanglement Entropy," JHEP 1201, 125 (2012) arXiv:1111.1023 [hep-th]].

[33] P. Dey and S. Roy, "Holographic entanglement entropy of the near horizon 1/4 BPS F-D $p$ bound states," arXiv:1208.1820 [hep-th].

[34] Y. Korovin, "Holographic Renormalization for Fermions in Real Time," arXiv:1107.0558 [hepth].

[35] U. Gursoy, E. Plauschinn, H. Stoof and S. Vandoren, "Holography and ARPES Sum-Rules," JHEP 1205, 018 (2012) arXiv:1112.5074 [hep-th]].

[36] M. Alishahiha, M. R. Mohammadi Mozaffar and A. Mollabashi, "Fermions on Lifshitz Background," Phys. Rev. D 86 (2012) 026002 arXiv:1201.1764 [hep-th]].

[37] J. -P. Wu and H. -B. Zeng, "Dynamic gap from holographic fermions in charged dilaton black branes," JHEP 1204, 068 (2012) arXiv:1201.2485 [hep-th]].

[38] U. Gursoy, V. Jacobs, E. Plauschinn, H. Stoof and S. Vandoren, "Lifshitz holography for undoped Weyl semimetals," arXiv:1209.2593 [hep-th].

[39] S. -S. Lee, "A Non-Fermi Liquid from a Charged Black Hole: A Critical Fermi Ball," Phys. Rev. D 79, 086006 (2009) arXiv:0809.3402 [hep-th]].

[40] H. Liu, J. McGreevy and D. Vegh, "Non-Fermi liquids from holography," Phys. Rev. D 83, 065029 (2011) arXiv:0903.2477 [hep-th]].

[41] M. Cubrovic, J. Zaanen and K. Schalm, "String Theory, Quantum Phase Transitions and the Emergent Fermi-Liquid," Science 325, 439 (2009) arXiv:0904.1993 [hep-th]].

[42] T. Faulkner, H. Liu, J. McGreevy and D. Vegh, "Emergent quantum criticality, Fermi surfaces, and AdS(2)," Phys. Rev. D 83, 125002 (2011) arXiv:0907.2694 [hep-th]].

[43] T. Faulkner, N. Iqbal, H. Liu, J. McGreevy and D. Vegh, "Strange metal transport realized by gauge/gravity duality," Science 329, 1043 (2010).

[44] S. A. Hartnoll, D. M. Hofman and D. Vegh, "Stellar spectroscopy: Fermions and holographic Lifshitz criticality," JHEP 1108, 096 (2011) arXiv:1105.3197 [hep-th]].

[45] M. Cubrovic, Y. Liu, K. Schalm, Y. -W. Sun and J. Zaanen, "Spectral probes of the holographic Fermi groundstate: dialing between the electron star and AdS Dirac hair," Phys. Rev. D 84, 086002 (2011) arXiv:1106.1798 [hep-th]]. 
[46] T. Faulkner, N. Iqbal, H. Liu, J. McGreevy and D. Vegh, "Holographic non-Fermi liquid fixed points," arXiv:1101.0597 [hep-th].

[47] J. N. Laia and D. Tong, "A Holographic Flat Band," JHEP 1111, 125 (2011) arXiv:1108.1381 [hep-th]].

[48] J. Bhattacharya, S. Cremonini and A. Sinkovics, "On the IR completion of geometries with hyperscaling violation," arXiv:1208.1752 [hep-th].

[49] N. Kundu, P. Narayan, N. Sircar and S. P. Trivedi, "Entangled Dilaton Dyons," arXiv:1208.2008 [hep-th].

[50] M. Alishahiha, M. R. M. Mozaffar and A. Mollabashi, "Holographic Aspects of Two-charged Dilatonic Black Hole in AdS5," arXiv:1208.2535 [hep-th].

[51] N. Iqbal, "Holography and Strongly Correlated Systems," Ph.D. thesis, MIT, June 2011.

[52] G. T. Horowitz, J. E. Santos and D. Tong, "Optical Conductivity with Holographic Lattices," JHEP 1207, 168 (2012) [1204.0519].

[53] M. Cvetic, M. J. Duff, P. Hoxha, J. T. Liu, H. Lu, J. X. Lu, R. Martinez-Acosta and C. N. Pope et al., "Embedding AdS black holes in ten-dimensions and eleven-dimensions," Nucl. Phys. B 558, 96 (1999) hep-th/9903214. 